\section{Saturated RISE Feedback Control for a Class of Second-Order Nonlinear Systems}

\author{
Nicholas Fischer, Zhen Kan, Rushikesh Kamalapurkar, and \\ Warren E. Dixon
}

\begin{abstract}
A saturated controller is developed for a class of uncertain, second-order, nonlinear systems which includes time-varying and nonlinearly parameterized functions with bounded disturbances using a continuous control law with smooth saturation functions. Based on the robust integral of the sign of the error (RISE) control methodology, the developed controller is able to utilize the benefits of high gain control strategies while guaranteeing saturation limits are not surpassed. The bounds on the control are known a priori and can be adjusted by changing the feedback gains. The saturated controller yields asymptotic tracking despite model uncertainty and added disturbances in the dynamics. Experimental results using a two-link robot manipulator demonstrate the performance of the developed controller.
\end{abstract}

Index Terms-Nonlinear control systems, RISE control, robust control, saturated control.

\section{INTRODUCTION}

Robust, high-gain controllers can be effective methods to compensate for nonlinear systems with unstructured parametric uncertainties and bounded disturbances. In general, robust control techniques (including all previous RISE methods) do not take into account the fact that the commanded input may require more actuation than is physically possible by the system (e.g., due to large initial condition offsets, an aggressive desired trajectory, or large perturbations). For example, the typical RISE structure uses a sufficiently large gain multiplied by an integral term, which could potentially exceed actuator capabilities under some conditions. Motivated by these issues, some efforts have focused on developing saturated controllers for the regulation problem (cf. [1]-[3]) and the more general tracking problem (cf. [4]-[10]). In [11], the authors developed an adaptive, full-state feedback controller to yield asymptotic tracking while compensating for unknown parametric uncertainties using multiple embedded hyperbolic saturation functions. The authors of [4] were able to extend the PID-based work of [1] to the tracking control problem by utilizing a general class of saturation functions to achieve a global uniform asymptotic tracking result for a linearly parameterizable system. The work was based on prior results in [12] and [5] which incorporated hyperbolic saturation functions into the saturated PD+ control strategy developed in [6]. The works of [4], [5], [12] exploit saturation-avoidance strategies. Anti-windup schemes have been developed in results such as [13] to compensate for saturation nonlinearities in nonlinear Euler-Lagrange systems using PID-like control structures. Results in [14] and

Manuscript received July 13, 2012; revised December 05, 2012, May 14, 2013, and October 06, 2013; accepted October 13, 2013. Date of publication October 23, 2013; date of current version March 20, 2014. This work was supported in part by the National Science Foundation under Awards 0901491, 1161260, 1217908, by ONR Grant N00014-13-1-0151, and by a contract with the AFRL Mathematical Modeling and Optimization Institute. Recommended by Associate Editor X. Chen.

The authors are with the Department of Mechanical and Aerospace Engineering, University of Florida, Gainesville, FL 32611 USA (e-mail: nic.r.fischer@gmail.com; kanzhen0322@ufl.edu; rkamalapurkar@ufl.edu; wdixon@ufl.edu).

Color versions of one or more of the figures in this paper are available online at http://ieeexplore.ieee.org.

Digital Object Identifier 10.1109/TAC.2013.2286913
[15] achieve global regulation of saturated nonlinear systems using a PID-like control structure and a passivity-based analysis. To compensate for uncertain dynamics and the evaluation of an unknown gravity term, the results in [7] includes an additional saturated integral term and uses energy shaping and damping injection methods to yield asymptotic tracking. More recently, a saturated PID controller was developed in [8] which uses sigmoidal functions to achieve global asymptotic regulation to a setpoint; however, it is unclear how the result can be extended to the tracking problem due to the inclusion of stationary values of an auxiliary signal. While each of the mentioned contributions developed saturated controllers with asymptotic tracking regulation, previous results have not been developed that include both uncertain dynamics and additive unmodeled disturbances. Control methods which include these considerations were developed by authors in [16] and [17] via saturated adaptive robust control (SARC) algorithms, which yield ultimately bounded tracking results.

Motivated to achieve an asymptotic result, Corradini, et al. developed a discontinuous saturated sliding mode controller [10] for linear plant models in the presence of bounded matched uncertainties. In [9], two control algorithms are developed for robust stabilization of spacecraft in the presence of control input saturation, parametric uncertainty, and external disturbances using a discontinuous variable structure control design. However, while each of these saturated robust techniques are able to address uncertain nonlinear systems with additive disturbances, the discontinuous nature of the results motivates the design of continuous saturated robust control techniques. Robust control designs utilizing nested saturation functions for uncertain feedforward nonlinear systems [18]-[20] can yield global asymptotic stability despite unmodeled dynamic disturbances.

Based on the preliminary work in [21] focused on Euler-Lagrange systems, this technical note focuses on a new RISE-based closed-loop error system development that consists of a saturated, continuous tracking controller for a class of uncertain, second-order nonlinear systems which includes time-varying and nonlinearly parameterized functions and unmodeled dynamic effects. The technical challenge presented by this objective is the need to introduce saturation bounds on the integral signum term while maintaining its functionality to implicitly learn the system disturbances. To achieve the result, a new auxiliary filter structure is designed using hyperbolic functions that work in tandem with the redesigned continuous saturated RISE-like control structure. While the controller is continuous, the closed-loop error system contains discontinuities which are examined through a differential inclusion framework. The resulting controller is bounded by the magnitude of an adjustable control gain, and yields asymptotic tracking.

\section{DYNAMIC SYSTEM}

Consider a class of second order MIMO nonlinear systems of the following form: ${ }^{1}$

$$
\ddot{x}=f(x, \dot{x}, t)+u(x, \dot{x}, t)+d(t)
$$

where $x, \dot{x} \in \mathbb{R}^{n}$ are the generalized system states, $u \in \mathbb{R}^{n}$ is the generalized control input, $f: \mathbb{R}^{n} \times \mathbb{R}^{n} \times[0, \infty) \rightarrow \mathbb{R}^{n}$ is an unknown nonlinear $C^{2}$ function, and $d \in \mathbb{R}^{n}$ denotes a generalized, sufficiently smooth, nonvanishing nonlinear disturbance (e.g., unmodeled effects).

The subsequent development is based on the assumption that $x$ and $\dot{x}$ are measurable outputs. Additionally, the following assumptions will be exploited.

\footnotetext{
${ }^{1}$ The result in this technical note can be extended to $n$ th-order nonlinear systems following a similar development to those presented in [22], [23].
} 
Assumption 1: The nonlinear disturbance term and its first two time derivatives (i.e., $d, \dot{d}, \ddot{d}$ ) exist and are bounded by known constants. ${ }^{2}$

Assumption 2: The desired trajectory $x_{d} \in \mathbb{R}^{n}$ is designed such that $x_{d}^{(i)} \in \mathbb{R}^{n}, \forall i=0,1, \ldots, 4$ exist and are bounded. ${ }^{3}$

Remark 1: To aid the subsequent control design and analysis, the vector $\operatorname{Tanh}(\cdot) \in \mathbb{R}^{n}$ and the matrix $\operatorname{Cosh}(\cdot) \in \mathbb{R}^{n \times n}$ are defined as

$$
\begin{aligned}
\operatorname{Tanh}(\xi) & \triangleq\left[\tanh \left(\xi_{1}\right), \ldots, \tanh \left(\xi_{n}\right)\right]^{T} \\
\operatorname{Cosh}(\xi) & \triangleq \operatorname{diag}\left\{\cosh \left(\xi_{1}\right), \ldots, \cosh \left(\xi_{n}\right)\right\}
\end{aligned}
$$

where $\xi=\left[\xi_{1}, \ldots, \xi_{n}\right]^{T} \in \mathbb{R}^{n}$. Based on these definitions, the following inequalities hold $\forall \xi \in \mathbb{R}^{n}[25]$ :

$$
\begin{gathered}
\|\xi\|^{2} \geq \sum_{i=1}^{n} \ln \left(\cosh \left(\xi_{i}\right)\right) \geq \frac{1}{2} \tanh ^{2}(\|\xi\|) \\
\|\xi\|>\|\operatorname{Tanh}(\xi)\|, \quad\|\operatorname{Tanh}(\xi)\|^{2} \geq \tanh ^{2}(\|\xi\|) \\
\xi^{T} \operatorname{Tanh}(\xi) \geq \operatorname{Tanh}^{T}(\xi) \operatorname{Tanh}(\xi) .
\end{gathered}
$$

Throughout the technical note, $\|\cdot\|$ denotes the standard Euclidean norm.

\section{Control OBJective}

The objective is to design an amplitude-limited, continuous controller which ensures the system state $x$ tracks a desired trajectory $x_{d}$. To quantify the control objective, a tracking error denoted by $e_{1} \in \mathbb{R}^{n}$ is defined as

$$
e_{1} \triangleq x_{d}-x
$$

Embedding the control in a bounded trigonometric term is an obvious way to limit the control authority below an a priori limit; however, by injecting these terms, difficulty arises in the closed-loop stability analysis. This challenge is exacerbated by the presence of integral control functions that are included to compensate for added disturbances as in this result. Motivated by these stability analysis complexities and through an iterative analysis procedure, two measurable filtered tracking errors are designed which include smooth saturation terms. Specifically, the filtered tracking errors $e_{2}, r \in \mathbb{R}^{n}$, are defined as

$$
\begin{gathered}
e_{2} \triangleq \dot{e}_{1}+\alpha_{1} \operatorname{Tanh}\left(e_{1}\right)+\operatorname{Tanh}\left(e_{f}\right) \\
r \triangleq \dot{e}_{2}+\alpha_{2} \operatorname{Tanh}\left(e_{2}\right)+\alpha_{3} e_{2}
\end{gathered}
$$

where $\alpha_{1}, \alpha_{2}, \alpha_{3} \in \mathbb{R}$ denote constant positive control gains, and $e_{f} \in$ $\mathbb{R}^{n}$ is an auxiliary signal whose dynamics are given by

$$
\dot{e}_{f} \triangleq \operatorname{Cosh}^{2}\left(e_{f}\right)\left\{-\gamma_{1} e_{2}+\operatorname{Tanh}\left(e_{1}\right)-\gamma_{2} \operatorname{Tanh}\left(e_{f}\right)\right\}
$$

and $\gamma_{1}, \gamma_{2} \in \mathbb{R}$ are constant positive control gains. The auxiliary signal $r$ is introduced to facilitate the stability analysis and is not used in the control design since the expression in (7) depends on the unmeasurable generalized state $\ddot{x}$. The structure of the error systems (and included auxiliary signals) is motivated by the need to inject and cancel terms in the subsequent stability analysis, and will become apparent in Section V.

${ }^{2}$ Many practical disturbance terms are continuous including friction (see [24]), wind disturbances, wave/ocean disturbances, unmodeled sufficiently smooth disturbances, etc.

${ }^{3}$ Many guidance and navigation applications utilize smooth, high-order differentiable desired trajectories. Curve fitting methods can also be used to generate sufficiently smooth time-varying trajectories.

\section{CONTROL DeVElopment}

An open-loop tracking error can be obtained by utilizing the filtered tracking error in (7) and substituting (1), (5), (6), and (8) to yield

$$
r=S-f_{d}+\ddot{x}_{d}-d-u(t)-\gamma_{1} e_{2}
$$

where the auxiliary function $S \in \mathbb{R}^{n}$ is defined as

$$
\begin{array}{r}
S \triangleq f_{d}-f-\gamma_{2} \operatorname{Tanh}\left(e_{f}\right)+\alpha_{1} \operatorname{Cosh}^{-2}\left(e_{1}\right)\left[e_{2}-\alpha_{1} \operatorname{Tanh}\left(e_{1}\right)\right. \\
\left.-\operatorname{Tanh}\left(e_{f}\right)\right]+\alpha_{2} \operatorname{Tanh}\left(e_{2}\right)+\alpha_{3} e_{2}+\operatorname{Tanh}\left(e_{1}\right)
\end{array}
$$

and $f_{d}=f\left(x_{d}, \dot{x}_{d}, t\right) \in \mathbb{R}^{n}$ is a desired trajectory dependent auxiliary term.

Based on the form of (9) and through an iterative stability analysis, the continuous controller, $u$, is designed as ${ }^{4}$

$$
u \triangleq \gamma_{1} \operatorname{Tanh}(v)
$$

where $v \in \mathbb{R}^{n}$ is a Filippov solution to the following differential equation

$$
\begin{aligned}
\dot{v}=\operatorname{Cosh}^{2}(v)\left[\alpha_{2} \operatorname{Tanh}\left(e_{2}\right)+\right. & \alpha_{3} e_{2}+\beta \operatorname{sgn}\left(e_{2}\right) \\
& \left.-\alpha_{1} \operatorname{Cosh}^{-2}\left(e_{1}\right) e_{2}+\gamma_{2} e_{2}\right]
\end{aligned}
$$

where $\beta \in \mathbb{R}$ is a positive constant control gain and $\operatorname{sgn}(\cdot)$ is defined $\forall \xi \in \mathbb{R}^{m}=\left[\begin{array}{llll}\xi_{1} & \xi_{2} & \ldots & \xi_{m}\end{array}\right]^{T}$ as $\operatorname{sgn}(\xi) \triangleq$ $\left[\operatorname{sgn}\left(\xi_{1}\right) \operatorname{sgn}\left(\xi_{2}\right) \ldots \operatorname{sgn}\left(\xi_{m}\right)\right]^{T}$.

In review of (5)-(10), the control strategy in (11) and (12) entails several components including the development of the filtered error systems in (6) and (7), which are composed of saturated hyperbolic tangent functions designed from the Lyapunov analysis. The motivation for the design of (8) stems from the need to inject a $-\gamma_{1} e_{2}$ signal into the closed-loop error system and to cancel terms in the analysis. Based on the stability analysis methods associated with the RISE control strategy (cf. [26], [27]), an extra derivative is applied to the closed-loop error system. The time derivative of (11) will include a $\operatorname{Cosh}^{-2}(v)$ term. The design of (12) is motivated by the desire to cancel the $\operatorname{Cosh}^{-2}(v)$ term, enabling the remaining terms to provide the desired feedback and cancel nonconstructive terms and disturbances as dictated by the subsequent stability analysis.

The closed-loop tracking error system can be developed by taking the time derivative of (9), and using the time derivative of (11) to yield

$$
\dot{r}=\tilde{N}+N_{d}-\gamma_{1} r-\gamma_{1} \beta \operatorname{sgn}\left(e_{2}\right)-\operatorname{Tanh}\left(e_{2}\right)-e_{2}
$$

where $\tilde{N} \in \mathbb{R}^{n}$ and $N_{d} \in \mathbb{R}^{n}$ are defined as

$$
\begin{aligned}
\tilde{N} \triangleq & \dot{S}+\gamma_{1} \alpha_{1} \operatorname{Cosh}^{-2}\left(e_{1}\right) e_{2} \\
& -\gamma_{1} \gamma_{2} e_{2}+\operatorname{Tanh}\left(e_{2}\right)+e_{2} \\
N_{d} \triangleq & \ddot{x}_{d}-\dot{f}_{d}-\dot{d} .
\end{aligned}
$$

The structure of (13) is motivated by the desire to segregate terms that can be upper bounded by state-dependent terms and terms that can be upper bounded by constants. By applying the Mean Value Theorem, an upper bound can be developed for the expression in (14) as

$$
\|\tilde{N}\| \leq \rho(\|w\|)\|w\|
$$

${ }^{4} \mathrm{An}$ important feature of the controller in (11) is its applicability to the case where constraints exist on the available control. Note that the control law is upper bounded by the adjustable control gain $\gamma_{1}$ as $\|u\| \leq \sqrt{n} \cdot \gamma_{1}$ where $n$ is the dimension of $u$. 
where the bounding function $\rho \in \mathbb{R}$ is a positive, strictly increasing function, ${ }^{5}$ and $w \in \mathbb{R}^{5 n}$ is defined as

$$
w \triangleq\left[\operatorname{Tanh}^{T}\left(e_{1}\right), e_{2}^{T}, r^{T}, \operatorname{Tanh}^{T}\left(e_{f}\right)\right]^{T} .
$$

From Assumptions 1 and 2, the following inequality can be developed based on the expression in (15):

$$
\left\|N_{d}\right\| \leq \zeta_{N_{d 1}}, \quad\left\|\dot{N}_{d}\right\| \leq \zeta_{N_{d 2}}
$$

where $\zeta_{N_{d 1}}, \zeta_{N_{d 2}} \in \mathbb{R}$, are known positive constants.

\section{STABILITY ANALYSIS}

To facilitate the stability analysis, let $\mathcal{D} \subseteq \mathbb{R}^{4 n+1}$ be the open and connected set defined as $\mathcal{D} \triangleq\left\{y \in \mathbb{R}^{4 n+1} \mid\|y\| \leq\right.$ in $\left.f\left(\rho^{-1}\left(\left[2 \sqrt{\lambda \gamma_{b}}, \infty\right)\right)\right)\right\}$, where $\lambda$ and $\gamma_{b}$ are subsequently defined. Let $z \in \mathbb{R}^{4 n}$ be defined as

$$
z \triangleq\left[e_{1}^{T}, e_{2}^{T}, r^{T}, \operatorname{Tanh}^{T}\left(e_{f}\right)\right]^{T}
$$

and $y \in \mathcal{D}$ be defined as

$$
y \triangleq\left[z^{T} \quad \sqrt{P}\right]^{T} .
$$

In (20), the auxiliary function $P \in \mathbb{R}$ is defined as a Filippov solution to the following differential equation

$$
\begin{gathered}
\dot{P} \triangleq-r^{T}\left(N_{d}-\beta \gamma_{1} \operatorname{sgn}\left(e_{2}\right)\right), \\
P\left(e_{2}\left(t_{0}\right), t_{0}\right)=\beta \gamma_{1} \sum_{i=1}^{n}\left|e_{2 i}\left(t_{0}\right)\right|-e_{2}\left(t_{0}\right)^{T} N_{d}\left(t_{0}\right)
\end{gathered}
$$

where the subscript $i=1,2, \ldots, n$ denotes the $i$-th element of the vector. Provided the sufficient condition for $\beta$ in (24) is satisfied, $P \geq$ 0 [21]. Let $V_{L}: \mathcal{D} \rightarrow \mathbb{R}$ be a positive-definite, locally Lipschitz, regular function defined as

$$
\begin{aligned}
V_{L} \triangleq \sum_{i=1}^{n} \ln \left(\cosh \left(e_{1 i}\right)\right) & +\sum_{i=1}^{n} \ln \left(\cosh \left(e_{2 i}\right)\right)+\frac{1}{2} e_{2}^{T} e_{2} \\
+ & \frac{1}{2} r^{T} r+\frac{1}{2} \operatorname{Tanh}^{T}\left(e_{f}\right) \operatorname{Tanh}\left(e_{f}\right)+P
\end{aligned}
$$

where $e_{1 i}$ and $e_{2 i}$ denote the $i$-th element of the vector $e_{1}$ and $e_{2}$, respectively. The Lyapunov function candidate in (22) satisfies the following inequalities:

$$
\phi_{1}(y) \leq V_{L}(y) \leq \phi_{2}(y), \quad \forall y \in \mathcal{D}
$$

Based on (4) and (22), the continuous, positive definite, strictly increasing functions $\phi_{1}, \phi_{2}: \mathcal{D} \rightarrow \mathbb{R}$ in (23) are defined as $\phi_{1} \triangleq$ $(1 / 2) \tanh ^{2}(\|y\|), \phi_{2} \triangleq(3 / 2)\|y\|^{2}$. Additionally, let $\mathcal{S} \subset \mathcal{D}$ denote a set defined as $\mathcal{S} \triangleq\left\{y \in \mathcal{D} \mid \rho\left(\sqrt{\phi_{2}(y)}\right)<2 \sqrt{\lambda \gamma_{b}}\right\}$.

\footnotetext{
${ }^{5}$ The proof in [28, App A] can be used to show that there exists a positive, nondecreasing bounding function for $\|\tilde{N}\|$. Any positive nondecreasing function can be upper bounded by a positive strictly increasing function, $\rho$.
}

Theorem 1: Given the dynamics in (1), the controller given by (11) and (12) ensures local ${ }^{6}$ asymptotic tracking in the sense that all Filippov solutions, such that $y\left(t_{0}\right) \in \mathcal{S}$, are bounded, and satisfy

$$
\left\|e_{1}\right\| \rightarrow 0 \text { as } t \rightarrow \infty
$$

provided the control gains are selected sufficiently large based on the initial conditions of the states and the following sufficient conditions:

$$
\begin{gathered}
\alpha_{1}>\frac{1}{2}, \quad \alpha_{2}>0, \quad \alpha_{3}>\frac{1}{2}+\frac{\gamma_{1}^{2} \zeta^{2}}{4}, \quad \gamma_{2}>\frac{1}{\zeta^{2}}, \\
\beta \gamma_{1}>\zeta_{N_{d 1}}+\frac{\zeta_{N_{d 2}}}{\alpha_{3}}, \quad 4 \lambda \gamma_{b}>\rho^{2}(\|w(0)\|)
\end{gathered}
$$

where $\lambda \in \mathbb{R}^{+}$is defined as $\lambda=\min \left\{\alpha_{1}-(1 / 2), 2 \alpha_{2}+\alpha_{3}, \alpha_{3}-\right.$ $\left.(1 / 2)-\left(\gamma_{1}^{2} \zeta^{2} / 4\right), \gamma_{2}-\left(1 / \zeta^{2}\right), \gamma_{a}\right\},\|w(0)\|$ denotes the initial conditions of the state, and $\gamma_{b}, \zeta \in \mathbb{R}$ are subsequently defined adjustable positive constants.

Proof: Let $y(t)$ for $t \in\left[t_{0}, \infty\right)$ denote a Filippov solution to the differential equation $\dot{y}=h_{3}(y, t)$ such that $y\left(t_{0}\right) \in \mathcal{S}$, where $h: \mathbb{R}^{4 n+1} \times \mathbb{R}>0 \rightarrow \mathbb{R}^{4 n+1}$ denotes the right hand side of the closed-loop error signals. Using Filippov's theory of differential inclusions [29], [30], the existence of solutions can be established for $\dot{y} \in K[h](y, t)$, where $K[h](y, t) \triangleq \bigcap_{\delta>0} \bigcap_{\mu S_{m}=0} \overline{c o} h\left(B(y, \delta) \backslash S_{m}, t\right)$, where $\bigcap_{\mu S_{m}=0}$ denotes the intersection of all sets $S_{m}$ of Lebesgue measure zero, $\overline{c o}$ denotes convex closure, and $B(y, \delta)=\left\{\varsigma \in \mathbb{R}^{n} \mid\|y-\varsigma\|<\delta\right\}$ [31], [32]. The time derivative of (22) along the Filippov trajectories exists almost everywhere (a.e.), i.e., for almost all $t \in\left[t_{0}, t_{f}\right]$, and $\dot{V}_{L} \stackrel{a . e .}{\in} \dot{\tilde{V}}_{L}$ where

$$
\dot{\tilde{V}}_{L}=\bigcap_{\xi \in \partial V_{L}(y, t)} \xi^{T} K\left[\dot{e}_{1}^{T} \dot{e}_{2}^{T} \dot{r}^{T} \operatorname{Cosh}^{-2}\left(e_{f}\right) \dot{e}_{f}^{T} \frac{1}{2} P^{-\frac{1}{2}} \dot{P} 1\right]^{T}
$$

and $\partial V_{L}$ is the generalized gradient of $V_{L}$ [33]. Since $V_{L}$ is a continuously differentiable

$$
\dot{\tilde{V}} \subset \nabla V_{L}^{T} K\left[\dot{e}_{1}^{T} \dot{e}_{2}^{T} \dot{r}^{T} \operatorname{Cosh}^{-2}\left(e_{f}\right) \dot{e}_{f}^{T} \frac{1}{2} P^{-\frac{1}{2}} \dot{P}\right]^{T}
$$

where

$$
\nabla V_{L} \triangleq\left[\operatorname{Tanh}^{T}\left(e_{1}\right),\left(\operatorname{Tanh}^{T}\left(e_{2}\right)+e_{2}^{T}\right), r^{T}, \operatorname{Tanh}^{T}\left(e_{f}\right), 2 P^{\frac{1}{2}}\right]^{T} .
$$

Using the calculus for $K[\cdot]$ from [32], and substituting (5)-(8), and (13) into (25), yields

$$
\begin{aligned}
\dot{\tilde{V}}_{L} \subset & r^{T}\left(\tilde{N}+N_{d}-\gamma_{1} r-\operatorname{Tanh}\left(e_{2}\right)-e_{2}\right)-r^{T}\left(\gamma_{1} \beta K\left[\operatorname{sgn}\left(e_{2}\right)\right]\right) \\
& +\operatorname{Tanh}^{T}\left(e_{1}\right)\left(e_{2}-\alpha_{1} \operatorname{Tanh}\left(e_{1}\right)-\operatorname{Tanh}\left(e_{f}\right)\right) \\
& +\operatorname{Tanh}^{T}\left(e_{2}\right)\left(r-\alpha_{2} \operatorname{Tanh}\left(e_{2}\right)-\alpha_{3} e_{2}\right) \\
& +e_{2}^{T}\left(r-\alpha_{2} \operatorname{Tanh}\left(e_{2}\right)-\alpha_{3} e_{2}\right) \\
& +\operatorname{Tanh}^{T}\left(e_{f}\right)\left(-\gamma_{1} e_{2}+\operatorname{Tanh}_{\left.\left(e_{1}\right)\right)}\right. \\
& +\operatorname{Tanh}^{T}\left(e_{f}\right)\left(-\gamma_{2} \operatorname{Tanh}\left(e_{f}\right)\right)+\dot{P}
\end{aligned}
$$

${ }^{6}$ For arbitrarily large initial conditions or arbitrarily large disturbances, the control gains required to satisfy the sufficient gain conditions in (24) may demand an input that is not physically deliverable by the system (i.e., the gain $\gamma_{1}$ may be required to be larger than the saturation limit of the actuator). Despite gain dependency on the system's initial conditions, this result does not satisfy the standard semi-global result because, under the consideration of input constraints, $\gamma_{b}$ cannot be arbitrarily increased and consequently the region of attraction cannot be arbitrarily enlarged to include all initial conditions. This outcome is not surprising from a physical perspective in the sense that such demands may yield cases where the actuation is insufficient to stabilize the system. 
where $K\left[\operatorname{sgn}\left(e_{2}\right)\right]=S G N\left(e_{2}\right)$ [32]. Substituting (21), (7), (16), and (18), the expression in (26) reduces to the scalar inequality

$$
\begin{aligned}
\dot{V}_{L} \stackrel{a . e .}{\leq} & -\alpha_{1}\left\|\operatorname{Tanh}\left(e_{1}\right)\right\|^{2}-\left(2 \alpha_{2}+\alpha_{3}\right)\left\|\operatorname{Tanh}\left(e_{2}\right)\right\|^{2} \\
& -\alpha_{3}\left\|e_{2}\right\|^{2}-\gamma_{2}\left\|\operatorname{Tanh}\left(e_{f}\right)\right\|^{2}-\gamma_{1}\|r\|^{2} \\
& +\rho\|w\|\|r\|+\left\|\operatorname{Tanh}\left(e_{1}\right)\right\|\left\|e_{2}\right\| \\
& +\gamma_{1}\left\|\operatorname{Tanh}\left(e_{f}\right)\right\|\left\|e_{2}\right\|
\end{aligned}
$$

where the set in (26) reduces to the scalar equality in (27) since the RHS is continuous a.e., i.e, the RHS is continuous except for the Lebesgue negligible set of times when $r^{T} \gamma_{1} \beta K\left[\operatorname{sgn}\left(e_{2}\right)\right]-$ $r^{T} \gamma_{1} \beta K\left[\operatorname{sgn}\left(e_{2}\right)\right] \neq 0$ [31], [34].7 Young's Inequality can be applied to select terms in (27) as

$$
\begin{gathered}
\left\|\operatorname{Tanh}\left(e_{1}\right)\right\|\left\|e_{2}\right\| \leq \frac{1}{2}\left\|\operatorname{Tanh}\left(e_{1}\right)\right\|^{2}+\frac{1}{2}\left\|e_{2}\right\|^{2} \\
\gamma_{1}\left\|\operatorname{Tanh}\left(e_{f}\right)\right\|\left\|e_{2}\right\| \leq \frac{1}{\zeta^{2}}\left\|\operatorname{Tanh}\left(e_{f}\right)\right\|^{2}+\frac{\gamma_{1}^{2} \zeta^{2}}{4}\left\|e_{2}\right\|^{2} .
\end{gathered}
$$

To facilitate the subsequent stability analysis, let $\gamma_{1}$ be selected as $\gamma_{1}=$ $\gamma_{a}+\gamma_{b}$, where $\gamma_{a}, \gamma_{b} \in \mathbb{R}$ are positive gain constants. Utilizing (28), completing the squares on $r$ and grouping terms, the expression in (27) can be upper bounded by

$$
\begin{aligned}
\dot{V}_{L} \stackrel{a . e .}{\leq} & -\left(\alpha_{1}-\frac{1}{2}\right)\left\|\operatorname{Tanh}\left(e_{1}\right)\right\|^{2}-\left(2 \alpha_{2}+\alpha_{3}\right)\left\|\operatorname{Tanh}\left(e_{2}\right)\right\|^{2} \\
& -\left(\alpha_{3}-\frac{1}{2}-\frac{\gamma_{1}^{2} \zeta^{2}}{4}\right)\left\|e_{2}\right\|^{2}-\left(\gamma_{2}-\frac{1}{\zeta^{2}}\right)\left\|\operatorname{Tanh}\left(e_{f}\right)\right\|^{2} \\
& -\gamma_{a}\|r\|^{2}+\frac{\rho^{2}(\|w\|)\|w\|^{2}}{4 \gamma_{b}} .
\end{aligned}
$$

Provided the sufficient conditions in (24) are satisfied, (17) and (19) can be used to conclude that

$$
\dot{V}_{L} \stackrel{\text { a.e. }}{\leq}-\phi_{3}(\|z\|) \leq-U(y)
$$

where $\phi_{3} \in \mathbb{R}$ is defined as $\phi_{3} \triangleq\left(\lambda-\left(\rho^{2}(\|w\|) / 4 \gamma_{b}\right)\right) \tanh ^{2}(\|z\|)$, $\lambda$ was defined in (24), and $U \triangleq c \tanh ^{2}(\|z\|) \forall y \subset \mathcal{D}$ is a continuous, positive semi-definite function for some positive constant $c \in \mathbb{R}$.

The inequalities in (23) and (30) can be used to show that $V_{L} \in \mathcal{L}_{\infty}$ in $\mathcal{D}$, hence, $e_{1}, e_{2}, r, \operatorname{Tanh}\left(e_{f}\right) \in \mathcal{L}_{\infty}$ in $\mathcal{D}$. From (2), $\operatorname{Tanh}\left(e_{1}\right), \operatorname{Tanh}\left(e_{2}\right) \in \mathcal{L}_{\infty}$ and from (6) and (7), $\dot{e}_{1}, \dot{e}_{2} \in \mathcal{L}_{\infty}$ in $\mathcal{D}$. From (11), $u \in \mathcal{L}_{\infty}$. From Assumption 2 and by utilizing the fact that $e_{1}, \dot{e}_{1} \in \mathcal{L}_{\infty}, q, \dot{q} \in \mathcal{L}_{\infty}$ in $\mathcal{D}$. From (17), $w \in \mathcal{L}_{\infty}$ in $\mathcal{D}$. Assumption 1, (13), (16) and (18) can be used to show that $\dot{r} \in \mathcal{L}_{\infty}$ in $\mathcal{D}$. Utilizing (8) and the fact that $e_{2} \in \mathcal{L}_{\infty}$ in $\mathcal{D}$, the product $\operatorname{Cosh}^{-2}\left(e_{f}\right) \dot{e}_{f} \in \mathcal{L}_{\infty}$ in $\mathcal{D}$. Thus, $\dot{z} \in \mathcal{L}_{\infty}$ in $\mathcal{D}$, and it can be shown that $z$ is uniformly continuous (UC) in $\mathcal{D}$. Since $z$ is UC, $\tanh (\|z\|)$ is UC. The definitions of $U$ and $z$ can be used to prove that $U$ is UC in $\mathcal{D}$.

Since $\rho$ and $\phi_{2}$ are strictly increasing functions, the region of attraction can be increased by increasing the gains. The fact that $\rho(0)=$ $c \neq 0$ for some $c \in \mathbb{R}_{+}$implies that in order to obtain a non-trivial region of attraction, the saturation bound $\gamma$ has to be large enough so that $2 \sqrt{\lambda \gamma_{b}}>c$. From (30), [36, Corollary 1] can be invoked to show that $\tanh (\|z\|) \rightarrow 0$ as $t \rightarrow \infty \forall y(0) \in \mathcal{S}$. Based on the definition of $z$ in (19), $\left\|e_{1}\right\| \rightarrow 0$ as $t \rightarrow \infty \forall y(0) \in \mathcal{S}$.

\footnotetext{
${ }^{7}$ The set of times $\Lambda \quad \stackrel{\Delta}{=} \quad\{t \quad[0, \infty)$ : $r(t)^{T} \gamma_{1} \beta K\left[\operatorname{sgn}\left(e_{2}(t)\right)\right]-r(t)^{T} \gamma_{1} \beta K\left[\operatorname{sgn}\left(e_{2}(t)\right)\right] \neq 0 \subset[0, \infty)$ is
equivalent to the set of times $\left\{t: e_{2}(t)=0 \wedge r(t) \neq 0\right\}$. From $(7)$, this set can also be represented by $\left\{t: e_{2}(t)=0 \wedge \dot{e}_{2}(t) \neq 0\right\}$. Provided $e_{2}(t)$ is continuously differentiable, it can be shown that the set of time instances $\left\{t: e_{2}(t)=0 \wedge \dot{e}_{2}(t) \neq 0\right\}$ is isolated, and thus, measure zero. This implies that the set $\Lambda$ is measure zero [35].
}

\section{EXPERIMENTAL RESULTS}

To examine the performance of the saturated RISE approach, the controller in (11) and (12) was implemented on a planar manipulator testbed. ${ }^{8}$ The manipulator can be modeled as an Euler-Lagrange system with the following dynamics

$$
M(q) \ddot{q}+V_{m}(q, \dot{q}) \dot{q}+F(\dot{q})=\tau
$$

where $M \in \mathbb{R}^{2 \times 2}$ denotes the inertia matrix, $V_{m} \in \mathbb{R}^{2 \times 2}$ denotes the unknown centripetal-Coriolis matrix, $F \in \mathbb{R}^{2}$ denotes a continuously differentiable friction model given in [24] as $F \triangleq M^{-1}(q)\left(\gamma_{1}\left(\tanh \left(\gamma_{2} \dot{q}\right)-\tanh \left(\gamma_{3} \dot{q}\right)\right)+\gamma_{4} \tanh \left(\gamma_{5} \dot{q}\right)+\gamma_{6} \dot{q}\right)$ where $\gamma_{i} \forall i=1,2, \ldots, 6$ are unknown positive constants that are related to friction coefficients. Additionally, in (31), $q, \dot{q}, \ddot{q} \in \mathbb{R}^{2}$ denote the link position, velocity and acceleration, and $\tau \in \mathbb{R}^{2}$ denotes the control torque. The Euler-Lagrange dynamics can be rewritten as

$$
\ddot{x}=f(x, \dot{x}, t)+\chi(x, \dot{x}, t)+u(x, t)+d(t)
$$

where $x=\left[q_{1}(t), q_{2}(t)\right]^{T}, f \triangleq-M^{-1}(q) V_{m}(q, \dot{q}) \dot{q}, u \triangleq$ $M^{-1}(q) \tau(t), \chi \triangleq M^{-1}(q) F(\dot{q})-M^{-1}\left(q_{d}\right) F\left(\dot{q}_{d}\right)$ and $d \triangleq$ $M^{-1}\left(q_{d}\right) F\left(\dot{q}_{d}\right)$. From (32) and [24], the friction disturbance $d$ satisfies Assumption 1. Other disturbances such as wind, ocean currents, etc. can also be shown to satisfy Assumption 1 for other electromechanical and aerospace systems. Moreover, given known bounds on the desired trajectory, and conservative upper bound estimates for the constant inertial parameters and friction constants, the inequalities in (24) can also be satisfied. The auxiliary term $\chi(x, \dot{x}, t)$ can be combined with $f(x, \dot{x}, t)$-like terms in $\tilde{N}(\cdot)$.

The control objective is to track a desired link trajectory, selected as $q_{d 1}=q_{d 2}=(45+60 \sin (2 t))\left(1-e^{-0.01 t^{3}}\right) \mathrm{deg}$. The initial conditions for the manipulator were selected a complete rotation away from the initial conditions of the desired trajectory as $q_{1}(0)=$ $360 \mathrm{deg}$ and $q_{2}(0)=-180 \mathrm{deg}$. The control torque was arbitrarily selected to be artificially limited (well-within the capabilities of the actuator) to $\left|\tau_{1}\right| \leq 60 \mathrm{Nm},\left|\tau_{2}\right| \leq 15 \mathrm{Nm}$. Specifically, the feedback gains for the proposed controller were selected as $\gamma_{1}=\operatorname{diag}(52,13)$, $\gamma_{2}=\operatorname{diag}(22,19), \beta=\operatorname{diag}(3.8,3.8), \alpha_{1}=\operatorname{diag}(6.2,6.0), \alpha_{2}=$ $\operatorname{diag}(8,11), \alpha_{3}=\operatorname{diag}(45,45), e_{f}(0,0)$ is selected as $e_{f}(0,0)=0$ and $v(0,0)$ is selected such that $u(0)=0 .{ }^{9}$

The performance of the saturated RISE control design was compared against two controllers available in literature: a classical PID controller with conditional integral clamping anti-windup [37] and an adaptive full-state feedback controller with bounded inputs [25]. Each controller was tuned to achieve the best possible performance, given the saturation bounds. Since each controller has a different structure, it is difficult to comment on the comparative nature of the gains which were implemented. Starting with the same large initial condition offset, the tracking errors of each controller are depicted in Fig. 1. The control torques for each controller are shown in Fig. 2 and each remain within the prescribed bounds.

The results show that the PID w/AW [37] achieves steady-state RMS errors of $8.3857 \mathrm{deg}$ and $1.4096 \mathrm{deg}$ for each joint, respectively. The

\footnotetext{
${ }^{8}$ The manipulator consists of a two-link direct drive revolute robot consisting of two aluminum links, mounted on $240.0 \mathrm{~N}-\mathrm{m}$ (base joint) and $20.0 \mathrm{~N}-\mathrm{m}$ (second joint) switched reluctance motors. The motor resolvers provide rotor position measurements with a resolution of 614400 pulses/revolution, and a standard backwards difference algorithm is used to numerically determine velocity from the encoder readings. Data acquisition and control implementation were performed in real-time using QNX at a frequency of $1.0 \mathrm{kHz}$.

${ }^{9}$ It is important to note that for the given Euler-Lagrange system, the implemented controller is $\tau=M(q) u$. Thus, the bound on the implemented control will include the (known) bound on the inertia matrix. For this experiment, the inertia matrix can be bounded by $\|M(q)\| \leq 1.15$.
} 


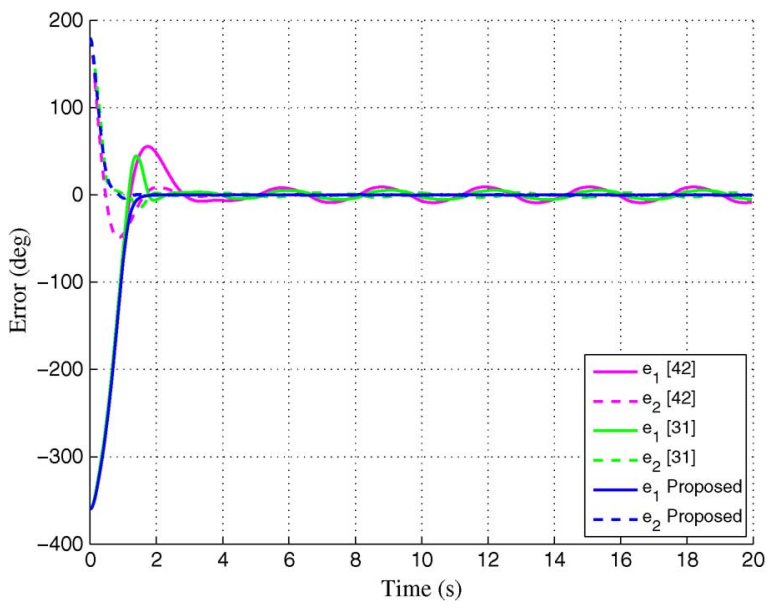

Fig. 1. Tracking errors versus time for (a) classical PID with integral clamping anti-windup, (b) adaptive full-state feedback controller, and (c) the proposed saturated RISE controller.
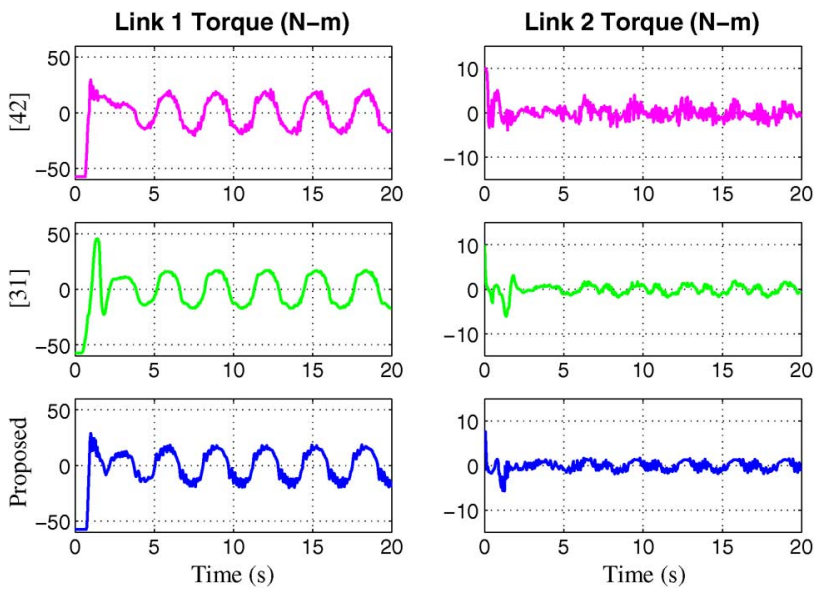

Fig. 2. Control torques versus time for (a) classical PID with integral clamping anti-windup, (b) adaptive full-state feedback controller, and (c) the proposed saturated RISE controller. Despite starting an entire revolution away from the desired trajectory, the controller only saturates at the preset artificial saturation limits briefly.

steady-state RMS torque for each joint was found to be $14.5630 \mathrm{Nm}$ and $1.8732 \mathrm{Nm}$, respectively. The Adaptive FSFB [25] steady-state RMS errors and torques for each joint were $3.8232 \mathrm{deg}, 1.6537 \mathrm{deg}$, 14.9367 Nm and 1.2360 Nm, respectively. The proposed Saturated RISE steady-state RMS errors and torques for each joint were 0.1607 deg, $0.2889 \mathrm{deg}, 14.3363 \mathrm{Nm}$ and $1.1883 \mathrm{Nm}$, respectively. The results illustrates that for comparable RMS torque values, the saturated RISE controller exhibits improved steady-state performance when compared to the other control designs.

\section{CONCLUSION}

A continuous saturated controller is developed for a class of uncertain nonlinear systems which includes time-varying and nonlinearly parameterized functions and additive bounded disturbances. The bound on the control is known a priori and can be adjusted by changing the feedback gains. The saturated controller is shown to guarantee local asymptotic tracking despite uncertainty in the dynamics using smooth hyperbolic functions. Experimental results using a two-link robot manipulator demonstrate the performance of the proposed controller.

\section{REFERENCES}

[1] A. Zavala-Rio and V. Santibanez, "A natural saturating extension of the PD-with-desired-gravity-compensation control law for robot manipulators with bounded inputs," IEEE Trans. Robot., vol. 23, no. 2, pp. 386-391, 2007.

[2] H. Yazarel, C. C. Cheah, and H. C. Liaw, "Adaptive SP-D control of a robotic manipulator in the presence of modeling error in a gravity regressor matrix: Theory and experiment," IEEE Trans. Robot. Autom., vol. 18, no. 3, pp. 373-379, 2002.

[3] V. Santibanez, R. Kelly, and M. Llama, "A novel global asymptotic stable set-point fuzzy controller with bounded torques for robot manipulators," IEEE Trans. Fuzzy Syst., vol. 13, no. 3, pp. 362-372, 2005.

[4] E. Aguinaga-Ruiz, A. Zavala-Rio, V. Santibanez, and F. Reyes, "Global trajectory tracking through static feedback for robot manipulators with bounded inputs," IEEE Trans. Control Syst. Technol., vol. 17, no. 4, pp. 934-944, 2009.

[5] J. Moreno-Valenzuela, V. Santibáñez, and R. Campa, "A class of OFT controllers for torque-saturated robot manipulators: Lyapunov stability and experimental evaluation," J. Intell. Rob. Syst., vol. 51, pp. 65-88, 2008.

[6] A. Loria and H. Nijmeijer, "Bounded output feedback tracking control of fully actuated Euler-Lagrange systems," Syst. Control Lett., vol. 33, pp. 151-161, Mar. 1998.

[7] J. Alvarez-Ramirez, V. Santibanez, and R. Campa, "Stability of robot manipulators under saturated PID compensation," IEEE Trans. Control Syst. Technol., vol. 16, no. 6, pp. 1333-1341, Nov. 2008.

[8] Y. Su, P. Muller, and C. Zheng, "Global asymptotic saturated PID control for robot manipulators," IEEE Trans. Control Syst. Technol., vol. 18, no. 6, pp. 1280-1288, 2010.

[9] J. D. Boskovic, S.-M. Li, and R. K. Mehra, "Robust adaptive variable structure control of spacecraft under control input saturation," J. Guid. Contr. Dynam., vol. 24, no. 1, pp. 14-22, 2001.

[10] M. Corradini, A. Cristofaro, and G. Orlando, "Robust stabilization of multi input plants with saturating actuators," IEEE Trans. Autom. Control, vol. 55, no. 2, pp. 419-425, Feb. 2010.

[11] W. E. Dixon, D. M. Dawson, F. Zhang, and E. Zergeroglu, "Global exponential tracking control of a mobile robot system via a pe condition," in Proc. IEEE Conf. Decis. Control, Phoenix, AZ, December 1999, pp. $4822-4827$.

[12] V. Santibanez and R. Kelly, "Global asymptotic stability of bounded output feedback tracking control for robot manipulators," in Proc. IEEE Conf. Decis. Control, 2001, vol. 2, pp. 1378-1379.

[13] F. Morabito, A. Teel, and L. Zaccarian, "Nonlinear antiwindup applied to Euler-Lagrange systems," IEEE Trans. Robotics Autom., vol. 20, no. 3, pp. 526-537, 2004.

[14] R. Gorez, "Globally stable PID-like control of mechanical systems," Syst. Control Lett., vol. 38, pp. 61-72, 1999.

[15] J. L. Meza, V. Santibanez, and V. Hernandez, "Saturated nonlinear PID global regulator for robot manipulators: Passivity based analysis," in Proc. IFAC World Congr., Prague, Czech Republic, 2005.

[16] Y. Hong and B. Yao, "A globally stable high-performance adaptive robust control algorithm with input saturation for precision motion control of linear motor drive systems," IEEE/ASME Trans. Mechatron., vol. 12, no. 2, pp. 198-207, 2007.

[17] L. Zhang, J. Xie, and D. Lu, "Adaptive robust control of one-link joint actuated by pneumatic artificial muscles," in Proc. Conf. BioInfo. Biomed. Eng., 2007, pp. 1185-1189.

[18] M. Arcak, A. Teel, and P. Kokotovic, "Robust nonlinear control of feedforward systems with unmodeled dynamics," Automatica, vol. 37, no. 2 , pp. 265-272, 2001

[19] L. Marconi and A. Isidori, "Robust global stabilization of a class of uncertain feedforward nonlinear systems," Syst. Control Lett., vol. 41, no. 4, pp. 281-290, 2000.

[20] G. Kaliora and A. Astolfi, "Nonlinear control of feedforward systems with bounded signals," IEEE Trans. Autom. Control, vol. 49, no. 11, pp. 1975-1990, Nov. 2004.

[21] N. Fischer, Z. Kan, and W. E. Dixon, "Saturated RISE feedback control for Euler-Lagrange systems," in Proc. American Control Conf., Montréal, Canada, Jun. 2012, pp. 244-249.

[22] N. Sharma, S. Bhasin, Q. Wang, and W. E. Dixon, "RISE-based adaptive control of a control affine uncertain nonlinear system with unknown state delays," IEEE Trans. Autom. Control, vol. 57, no. 1, pp. 255-259, Jan. 2012. 
[23] S. Bhasin, N. Sharma, P. Patre, and W. E. Dixon, "Asymptotic tracking by a reinforcement learning-based adaptive critic controller," J. Control Theory and App., vol. 9, no. 3, pp. 400-409, 2011.

[24] C. Makkar, G. Hu, W. G. Sawyer, and W. E. Dixon, "Lyapunov-based tracking control in the presence of uncertain nonlinear parameterizable friction," IEEE Trans. Autom. Control, vol. 52, pp. 1988-1994, 2007

[25] W. E. Dixon, M. S. de Queiroz, D. M. Dawson, and F. Zhang, "Tracking control of robot manipulators with bounded torque inputs," Robotica, vol. 17, pp. 121-129, 1999.

[26] P. M. Patre, W. Mackunis, C. Makkar, and W. E. Dixon, "Asymptotic tracking for systems with structured and unstructured uncertainties," IEEE Trans. Control Syst. Technol., vol. 16, pp. 373-379, 2008.

[27] P. M. Patre, W. MacKunis, K. Kaiser, and W. E. Dixon, "Asymptotic tracking for uncertain dynamic systems via a multilayer neural network feedforward and RISE feedback control structure," IEEE Trans. Autom, Control, vol. 53, no. 9, pp. 2180-2185, Sep. 2008.

[28] M. de Queiroz, J. Hu, D. Dawson, T. Burg, and S. Donepudi, “Adaptive position/force control of robot manipulators without velocity measurements: Theory and experimentation," IEEE Trans. Syst. Man Cybern., vol. 27-B, no. 5, pp. 796-809, 1997.

[29] A. F. Filippov, Differential Equations With Discontinuous Right-Hand Sides. Norwell, MA: Kluwer, 1988.

[30] J. P. Aubin and H. Frankowska, Set-Valued Analysis. Basel, Switzerland: Birkhäuser, 2008.

[31] D. Shevitz and B. Paden, "Lyapunov stability theory of nonsmooth systems," IEEE Trans. Autom. Control, vol. 39, no. 9, pp. 1910-1914, Sep. 1994.

[32] B. Paden and S. Sastry, "A calculus for computing Filippov's differential inclusion with application to the variable structure control of robot manipulators," IEEE Trans. Circuits Syst., vol. CAS-34, no. 1, pp. 73-82, 1987.

[33] F. H. Clarke, Optimization and Nonsmooth Analysis. Philadelphia, PA: SIAM, 1990.

[34] R. Leine and N. van de Wouw, "Non-smooth dynamical systems," in Stability and Convergence of Mechanical Systems With Unilateral Constraints, ser. Lecture Notes in Applied and Computational Mechanics. Berlin/Heidelberg: Springer, 2008, vol. 36, pp. 59-77.

[35] R. Kamalapurkar, J. Klotz, R. Downey, and W. E. Dixon, 2013 , Supporting Lemmas for RISE-Based Control Methods arXiv:1306. 3432.

[36] N. Fischer, R. Kamalapurkar, and W. E. Dixon, "LaSalle-Yoshizawa corollaries for nonsmooth systems," IEEE Trans. Automat. Control, vol. 58, no. 9, pp. 2333-2338, 2013.

[37] K. S. Walgama, S. Ronnback, and J. Sternby, "Generalization of conditioning technique for anti-windup compensators," Proc. IEE Control Theory Appl., vol. 139, no. 2, pp. 109-118, 1992.

\section{Sensitivity Reduction by Stable Controllers for MIMO Infinite Dimensional Systems via the Tangential Nevanlinna-Pick Interpolation}

\author{
Masashi Wakaiki, Student Member, IEEE, \\ Yutaka Yamamoto, Fellow, IEEE, and \\ Hitay Özbay, Senior Member, IEEE
}

\begin{abstract}
We study the problem of finding a stable stabilizing controller that satisfies a desired sensitivity level for an MIMO infinite dimensional system. The systems we consider have finitely many simple transmission zeros in $\overline{\mathbb{C}}_{+}$, but they are allowed to possess infinitely many poles in $\mathbb{C}_{+}$. We compute both upper and lower bounds of the minimum sensitivity achievable by a stable controller via the tangential Nevanlinna-Pick interpolation. We also obtain stable controllers attaining such an upper bound. To illustrate the results, we discuss a repetitive control system as an application of the proposed method.
\end{abstract}

Index Terms $-\mathcal{H}^{\infty}$ control, infinite dimensional systems, strong stabilization, tangential interpolation.

\section{INTRODUCTION}

The purpose of this note is to find stable controllers achieving a desired sensitivity level for MIMO infinite dimensional systems. Let us first note that even for stable plants, $\mathcal{H}^{\infty}$ optimization may produce unstable controllers. However, such controllers have difficulties with robustness and hardware implementation. Indeed, an unstable controller can lead to instability of the closed-loop system if a component such as a sensor or an actuator fails [1] or saturates [2]. See also [3]-[5] for theoretical and practical significance of stabilization by a stable controller. Applications of stable $\mathcal{H}^{\infty}$ controllers can be found in flexible structures [6], DC servo motors [7], data-communication networks [8], etc.

For SISO infinite dimensional systems, the Nevanlinna-Pick interpolation [9], [10] enables us to design stable controllers providing the minimum sensitivity [11] or robust stability [12]. The point of this approach is that a stable controller stabilizes the plant if and only if a unit element in $\mathcal{H}^{\infty}$ satisfies certain interpolation conditions at the unstable zeros of the plant. On the other hand, for MIMO infinite dimensional systems, the stable $\mathcal{H}^{\infty}$ controller design problem is still largely open. This is due to the difficulty of multivariable zeros.

We have studied sensitivity reduction by a stable controller for MIMO systems with infinitely many unstable poles in [13]. We have shown there that the matrix-valued Nevanlinna-Pick interpolation [3], [14] gives a sufficient condition and also a necessary condition for this problem. However the results in [13] are subject to the rather stringent assumption that all unstable zeros of the plant be blocking

Manuscript received May 08, 2013; revised August 23, 2013 and September 11, 2013; accepted September 29, 2013. Date of publication October 17, 2013; date of current version March 20,2014. This paper was presented in part at the 51st IEEE Conference on Decision and Control. Recommended by Associate Editor L. Mirkin.

M. Wakaiki and Y. Yamamoto are with the Department of Applied Analysis and Complex Dynamical Systems, Graduate School of Informatics, Kyoto University, Kyoto 606-8501, Japan (e-mail: wakaiki@acs.i.kyoto-u.ac.jp; yy@i. kyoto-u.ac.jp).

H. Özbay is with the Department of Electrical and Electronics Engineering, Bilkent University, Bilkent, Ankara TR-06800,Turkey (e-mail: hitay@bilkent. edu.tr).

Color versions of one or more of the figures in this paper are available online at $\mathrm{http}: / /$ ieeexplore.ieee.org.

Digital Object Identifier 10.1109/TAC.2013.2285788 